\title{
Research on the Problems and Countermeasures of English Translation based on Aesthetics and the Perspective of Cultural Equivalence
}

\author{
Wu Fei ${ }^{1}$ \\ ${ }^{1}$ TianJin Maritime College TianJin 300350 China
}

\begin{abstract}
In this paper, we conduct research on the problems and countermeasures of English translation based on aesthetics and the perspective of cultural equivalence. Semantic information equivalence including the deep and shallow levels of semantic information equivalence that is both the surface layer of language meaning and deep meaning. Behave extremely incisively and vividly is English translation. To improve the general English translation of science and technology aesthetics, should from the translator's aesthetic consciousness and abilities to express. Our research provides the novel paragdim for English translation which will be meaningful.
\end{abstract}

Keywords: English Translation; Cultural Equivalence; Problems and Countermeasures.

\section{Introduction}

English translation as a professional skill, often needs to face more complicated than daily spoken English and written expression form and content, on the expression of language translation also needs to meet the requirements of higher, and in the translation process will often meet specialized vocabulary or technical which requires a translation personnel to consult or learn relevant background knowledge, understand the cultural background of western countries and the special situation. So relatively ordinary English speaking English translation has higher professional requirements, the goal of translation is expounded by the language will be a kind of language to express ideas through transformation to meet another language background people read or understand. Since birth, English has become an expression activities have been conducted, it is for English has a huge impact on the development of national culture and language, and for other language also have certain influences on the development of the country. For the conversion between English and other languages, the basic communication plays a big role in promoting, on the development of English and other language also has a certain role in promoting. In the process of English translation for English and other languages of the historical and cultural background to have a comprehensive understanding, to the same period under different reading people also want to have a certain understanding, speaking, reading and writing habits. In business English translation, the transformation of parts of speech is the expression a great skill. In Chinese and English two languages are heavier we found that the two languages is adjectives, verbs, nouns, such as has the same syntax similarity, but regional differences of China and the United States led to the two languages has its special expression and meaning. In addition, with combination of grammar in English and Chinese languages, there is a big difference between difference is also evident in terms of semantic segmentation. In the actual application of vocabulary more are world apart, significant difference. Therefore, in the real translation, we can't always use some type of vocabulary to corresponding single words all the time and condition [1].

Semantic information equivalence including the deep and shallow levels of semantic information equivalence that is both the surface layer of language meaning and deep meaning, 
when translation should comply with the principle of reciprocity which makes the translation more accurate. Tend to have a certain cultural background, the language history and culture. Except business contract, this is because the business contract has legal significance, must use formal language and we cannot adopt indirect contains the expression of language and culture background, also not to extension. Because has the huge cultural differences between different countries and nations, and so on the English translation, it is very important for the cultural background of mining [2]. To correctly grasp the differences of English translator in the translation before you know the culture between the country and the nation, according to the cultural background to a certain degree of change, make the translation more accurate. In addition, the English translation should also respect the reader's real reflection, the ultimate goal of English translation and English readers reaction equivalence is Chinese readers. The results in English translation, the translation should achieve the same as the original language expression effect. People from different cultural backgrounds communicate is the process of cross-cultural communication. Different nations have different historical background, customs, local conditions and customs, cultural traditions, therefore, to engage in international business translators must understand the grasp the domestic and foreign national cultural differences, and try to eradicate the differences in the process of translation, at the same time to find the exact words in the target language and make an exotic culture reappear in the target language.

Phonetic unit is a basic element of voice. English pronunciation is divided into two kinds, one is consonant. The first letter is a vowel. Music analysis to the smallest unit of sound system is also the consonants and vowels. Actually, people in the creative use of language are always putting the audio language sequence one thousand to compose the 'music'. As far as possible harmonious rhythm, this is the voice of "music". This is most obvious in the English language. Behave extremely incisively and vividly is English translation. To improve the general English translation of science and technology aesthetics, should from the translator's aesthetic consciousness and abilities to express. Translators to understand and express determines the quality and characteristics of translation. First start with the details of life cultivation of the aesthetic consciousness of the translator, the beauty of language accurate, concise and logical for the accumulation of the certain, then strengthen the cultivation of the Chinese expression ability of the translator which make its ability to accurately express the meaning.

In this paper, we conduct research on the problems and countermeasures of English translation based on aesthetics and the perspective of cultural equivalence. English translation as a means of a new form of media and information conversion tool, in today's international trade plays a more and more important role. It is not only an information conversion tool, or human bridge of intercultural communication, for human development, economic globalization which makes a great contribution. In the following sections, we will research on the issues in detail.

\section{Our Proposed Methodology}

The Perspective of Cultural Equivalence. Language is carrier of culture it plays a very important role on the culture. Inter-lingual translation is not only the exchange of two languages, also asked two kinds of culture transmission. The translation of the original which must be accompanied with the code, it is often required in phonological, lexical, syntactic and discourse levels, such as a series of adjustment. For example, Nike trademark can make English country people associated with the victory, auspicious, because Nike is a word in Greek mythology is the goddess of victory. Nike sports goods as trademark, we can put it, "Nike", 
suggests that the Nike brand sporting goods over a long period, durable which can use the Nike brand sporting goods the enemy in the game. Each nation has its unique culture. Eastern and western people have different philosophy and culture. Therefore, cultural differences will inevitably exist. In addition, the difference reason lies in the east and west national geography, history, different local conditions and customs, etc. [3]. It is the presence of these cultural differences make the translatability of cultural translation is restricted.

With international business English translation must pay special attention to these differences, must be in foreign culture and native culture find a suit a point. If the suit a point was the interaction of the two cultures, that is the most ideal. The principles points of translation could be summarized as the follows. (1) Words in business English translation, the translation must be the general rental terms of equivalence, which is the translation of business English should use fixed professional vocabulary, demonstrate its professionalism. Usually, the business English professional term can only be a single interpretation, there is no synonym replacement, this requires that business English translators should master a lot of English professional knowledge, and to understand the business negotiation process. Once appear translation errors, it is also easy to cause misunderstanding or unnecessary trouble. But business English translation method, literal translation method can be used or language equivalence translation method. (2) Sentence is an important part of the article, in addition to the vocabulary of substandard, statements of translation should also follow the equivalence theory. First the original text to reflect the cultural background, then the structure of the original and some words according to the actual need to make appropriate changes, but we can't change the basic meaning of sentences. Peer requested translation can accurately express the meaning of the article or language. (3) Based on the theory of functional equivalence in business English, English discourse language translation requires the translator must first complete the functional equivalence on the words and sentences. The translation of that is to say, to analyze the characteristics of the statements and statements contained in the cultural background. So in translation, we first must carry on the analysis of words and statements. Then under the guidance of Nida's functional theory and based on the general and overall article expresses deep meaning and style and the special articles.

In the process of two kinds of language and culture contrast, we must make reasonable adjustments to the cultural differences in translation. The original alleged object seems to be meaningless. The alleged object may cause readers misunderstanding. Target language semantics "overload" and let the reader to understand. As a cultural carrier of language, must have rich cultural background, which makes some words in the English can only be found in the part of the Chinese corresponding word, even there is no corresponding language. Its cultural heaviest load, when the translation should cultural adjustment. Chinese-English word referential translation in general there are four methods namely literal translation method. Literal translation method is a commonly used translation method. Translation is to make the important way of two kinds of culture and language fusion as a business English translation has a certain complexity and purpose. It puts forward higher requirements on business English translators, this paper analyzes the application of the theory of functional equivalence in business English translation and the purpose is to improve business efficiency and the accuracy of translation which will promote the translation activities.

The Skills of English Translation. For the translator to English to Chinese sometimes nearly as much as the original English writing difficulty, some professional or special vocabulary to express more appropriate often 
require huge energy and cost, both in English and Chinese expressions with ever-changing difference, translation not only in words to fit to the original, but also conform to the character of the place and time, the smooth and accurate. Professional English translation needs to be able to face the problem of myriad professional environment, there are a lot of words with multiple meanings, or even just expressed by the thumbnail of the form, so it is hard to find reference in ordinary dictionaries, so this kind of vocabulary translation accuracy is very important and although some words are ordinary literally spelling, but embodied in the special environment of professional meaning is not the same which need the translator more profound understanding of the professional environment. In English, especially in the process of business English translation, the accuracy is always our pursuit of the first target. This requests the translator to translate business language in the process of should pay attention to ponder and the expression of the concept of vocabulary, especially pay attention to some numerical statement and unit of the mark. English is there is a big difference with other forms of language expression, it is the content of higher precision and loyalty, which also calls for the use of English translation skills in the process of pay more attention to practical.

How to successfully improve the practical skills in English translation could be summarized as the follows. (1) Choose the exact words, to ensure the integrity of the translation in business English translation work and contract translation is a very important work. It is capable of legally binding, for the two sides signed must be strictly observed. Therefore in the process of the translation of business contracts the extra careful, pay attention to the choice of words. (2) Reasonable increase or decrease in vocabulary, strengthen the practicality should be clear, in English translation practice, how to reasonable and appropriate vocabulary is a must to master the skills of increase or decrease. To grasp such a degree that requires the context of the original repeated analysis and thorough understanding, straighten out the author's thinking logic, grasp the characteristics of syntax and language environment and habits, improve the flexibility in the process of translating, but does not allow for some original language environment to express such a mean words add, don't need the words in the translated text abridge, ensure the smoothness of the translation, does not damage the integrity of the original at the same time, guarantee the information equivalence. (3) Be flexible transformation in the process of translation in business English translation, the transformation of work to do is to express the way of proper transformation and the change of part of speech. Generally speaking, Chinese and English expression and habits are there was a big difference. So a lot of translators in the process of translation is one of the biggest difficulties can't be the same part of speech and expression.

The Aesthetics and English Translation. As the edge discipline of translation studies, translation aesthetics is by using the basic principle of aesthetics and linguistics to explore the problem of inter-lingual translation aesthetics in translation. In this perspective, translation is the aesthetic main body through aesthetic medium (the aesthetic consciousness of the translator) converts the aesthetic object to another a kind of aesthetic activity of aesthetic object. Based on the basic theory of translation aesthetics, translation is the translator of the aesthetic experience produced a kind of aesthetic feeling of the original setting, then the process of expressed in another language. Visible, translation aesthetics is discussed the beauty of the original and the translation and the translation process. Language is the primary factor in the literature, in order to create accurate, distinct, vivid, give a person a kind of pleasant, joyfully Yi in aesthetic feeling, author intends to show the formal beauty of the language, often used to produce some kind of rhetoric or aesthetic effects. The aesthetic function of language is made up of 
epic, visual and audible beauty performance, language form in the physical form of the language of sounds and written symbols above characteristics. To guide translation is to make the translation and the original with aesthetics is the effective means to achieve the same effect, the process of translation is the process of aesthetic orientation.

In the English translation of euphemism is one kind of unique aesthetic value. Tactfully beauty which means on the wording to be honest polite, don't be aggressive in the language. In spite of the practical activities, fierce competition behavior is more common, but gentle communication is also essential. Polite not only to win the respect of others, but could effectively relieve tense negotiations atmosphere. Therefore, in the actual text translation, translators should pay attention to the cultural difference between the two languages, strive for polite through the translation of the original, in order to better meet the requirements of business English translation aesthetics which will hold the special meaning and necessity.

\section{Conclusion}

In this paper, we conduct research on the problems and countermeasures of English translation based on aesthetics and the perspective of cultural equivalence. English translation has its own unique characteristics, in its text translation, we should not only the content of the text information on time as requests complete transformation, transmission, and achieve the conversion of beauty. Based on the complexity and diversity of the English text type at the same time, we also targeted according to different text types adopt different ways of translation, only then can achieve the purpose of improving the quality of English translation. In the future, we plan to combine more literature review to optimize the current translation pattern to achieve better performance.

\section{References}

[1] Wang F. Business English Translation from the Aesthetic Perspective[J]. Journal of Ningbo University of Technology, 2013.

[2] Liuyue H U, School F L, University Z S. On Principles of the English Translation of Chinese Dialects: With Shaoxing Dialect as an Example[J]. Journal of Zhejiang International Studies.

[3] Zhai X L. Application on A Bite of China in College English Translation Teaching[J]. Overseas $\quad 2014$. 